\title{
ANÁLISIS DEL SISTEMA PENITENCIARIO FRENTE A LA REINSERCIÓN SOCIAL EN MÉXICO
}

\author{
Lorena Martínez Martínez ${ }^{1}$ \\ José Cruz Guzmán Díaz
}

\section{Resumen}

El Sistema Penitenciario es un apartado sumamente importante dentro de la Seguridad Pública, puesto que es la última etapa donde una persona después de haber cometido un delito y sujeta a un proceso penal es condenado a cumplir una sentencia por encontrársele como responsable de la comisión de un ilícito, se somete a la pena de prisión misma que tiene como principal objetivo la Reinserción Social, la cual está a cargo de instituciones, funcionarios que tienen como fin cumplir con este proceso. Ahora bien derivado de algunas situaciones nuestro Sistema Penal en junio de dos mil ocho se reforma de ser Mixto a Acusatorio Adversarial, sufre esta modificación en virtud de que como venía operando el Sistema no se alcanzaban a cubrir los resultados óptimos, es decir, estaban perdiendo credibilidad las instituciones encargadas de impartir y administrar justicia, el rezago judicial que imperaba en México ya era demasiado, la corrupción, y con ello algunas violaciones a los derechos humanos tanto de las personas en prisión preventiva como de las personas que cumplían propiamente una pena de prisión, por tanto nos planteamos el objetivo de analizar el sistema penitenciario frente a la reinserción social en México, a través de una investigación jurídica, dogmática, con un enfoque cualitativo y descriptiva, que arrojo como conclusión que si

$1 \quad$ Lorena Martínez Martínez, Doctora en Derecho, Profesor de Tiempo Completo del Centro Universitario del Sur de la Universidad de Guadalajara, Jalisco. México. Investigador del Observatorio Ciudadano de Cultura de la Legalidad y Sistema de Justicia en la misma institución educativa. Email: lorenamm@cusur.udg.mx

2 José Cruz Guzmán Díaz, Doctor en Derecho, Coordinador de la Maestría en Derecho y Profesor de Tiempo Completo del Centro Universitario del Sur de la Universidad de Guadalajara, Jalisco. México. Investigador del Observatorio Ciudadano de Cultura de la Legalidad y Sistema de Justicia en la misma institución educativa. Email: joseg@ cusur.udg.mx 
bien es todo un reto, se tendrá éxito si cada uno de las instituciones, operadores del sistema y personal penitenciario trabajan en conjunto se logrará la Reinserción Social anhelada.

Palabras clave: Derecho penal, sistema acusatorio y reinserción social.

\section{Abstract}

The prison system is an extremely important part of the public security system, since it is the last stage where a person, after committing a crime and being subject to criminal proceedings, is sentenced to serve a sentence for being found responsible for the commission of an illicit act. However, as a result of some situations, our Criminal System was reformed from being a Mixed to an Adversarial Accusatory in June 2008. This change was made because the system had been operating but had not achieved optimum results, i.e. the institutions in charge of imparting and administering justice were losing credibility, the judicial backlog that prevailed in our country was already too great, corruption, and with it some violations of the human rights of both persons in preventive detention and persons serving a prison sentence, therefore, we set ourselves the goal of analyzing the prison system in relation to social reinsertion in Mexico, through legal and dogmatic research, with a qualitative and descriptive focus, which concluded that although it is a challenge, it will be successful if each of the institutions, system operators and prison staff work together to achieve the desired social reinsertion.

Keywords: Criminal law, accusatory system and social reintegration. 


\section{Introducción}

El problema de la sobrepoblación en centros penitenciarios, los motines en centros carcelarios, entre otras; viendo la problemática que se ve la necesidad de hacer adecuaciones al Sistema Penal, se realiza esta modificación con fecha 18 de junio de 2008; se establece una prórroga para que en término de ocho años posteriores a esta, todos los Estados de la República Mexicana implementaran el nuevo Sistema Penal Acusatorio, ahora bien en este caso cada uno de ellos se vio en la necesidad en primer lugar de capacitar a los operadores del sistema y realizar las modificaciones en las instalaciones, ya que no se contaba con los espacios ni con las Salas para la realización de las audiencias; en esta reforma penal se modificaron los artículos 15,16,17,18,19,20 y 21 de nuestra Constitución Política de los Estados Unidos Mexicanos, mediante el Decreto publicado en el Diario Oficial de la Federación de fecha 18 de junio de 2008, se reforman y adicionan diversas disposiciones de la Constitución Política de los Estados Unidos Mexicanos.

Por lo que en este presente trabajo nos enfocaremos en especial a los artículo 18 y 21 constitucional en donde se establece cuáles son las bases del Sistema Penitenciario en nuestro País, así como algunas especificaciones de cómo debe ser su funcionamiento para lograr el objetivo en específico como lo es la Reinserción Social y sobre todo la nueva figura del Juez de Ejecución de Penas en donde la finalidad es de que esta autoridad judicial, sea la responsable de la Ejecución Penal, caso en particular que en nuestro país no existía sino que la vigilancia y seguimiento de la Ejecución Penal estaba a cargo del Poder Ejecutivo, así como el surgimiento de la Ley Nacional de Ejecución de Penas.

Se toman en cuenta estos aspectos de reforma pues de ellos puedo hacer un análisis en que momento nos encontramos después de estas reformas en relación a si la Reinserción Social con este enfoque que se da, ha permitido que se cumpla o que factores no han permitido que se cumpla el objetivo del Sistema Penitenciario en México. 


\section{Legislación mexicana aplicable al sistema penitenciario actual - La Reforma Constitucional de los Artículos 18 y 21}

Dentro de la exposición de motivos para la reforma constitucional en la que cada uno de los Grupos Parlamentarios presentaron las propuestas de reforma a fin de que fueran consideradas y aprobadas, que se presentó mismos que se tomaron en cuenta para la realización de la reforma fueron los siguientes puntos, partiendo de que las prisiones en México se han visto como un gasto indeseable, y no se les ha tomado la importancia que estas debieran tener, motivo por el cual se ha provocado que en las prisiones se conviertan en lugares donde se violan los derechos humanos de los reclusos. A pesar de que por mandato constitucional se establece que estas personas que se encuentran privadas de su libertad tengan acceso a la educación al trabajo y por lo tanto no existan las condiciones necesarias para que los reclusos ejerzan estos derechos.

- Una de las cuestiones que ha causado alarma es que dentro de los centros penitenciarios no se cuentan con un servicio de salud digno que se les pueda ofrecer a las personas privadas de la libertad, en muchos de ellos no se cuenta ni siquiera con el personal indispensable para atender alguna emergencia que se pudiera suscitar dentro de estos establecimientos, cuanto menos de los instrumentos necesarios para dar una consulta, el medicamento para dar un tratamiento o instalaciones adecuadas para dar una consulta médica.

- La alimentación por otra parte es precaria y no es la adecuada para la población penitenciaria, en muchos de los establecimientos no son reguladas las porciones y en muchas de ellas no cuenta con la vigilancia de sanidad en cuanto a la preparación de los alimentos.

- Por otra parte, las instalaciones en su mayoría son viejas e insalubres y deterioradas, sin dejar de largo la sobrepoblación que existe en la mayoría de las instituciones penitenciarias en el País.

- El hacinamiento obstaculiza realizar las funciones esenciales del sistema penitenciario como la salud, la seguridad, el régimen de visitas, entre otras, limitado con ello se realicen las funciones adecuadamente tales como atender las adicciones que padecen 
los reclusos, la recreación, así como la visita íntima. Por tal motivo se violan los derechos fundamentales tanto de las personas privadas de la libertad como de los empleados quienes trabajan en situaciones riesgosas y evitan la actuación de las funciones propias de cada uno de ellos.

Dicha reforma tenía como finalidad el dar un giro al funcionamiento del sistema penitenciario, de tal forma que la ejecución de penas no estuviera bajo el control absoluto del Poder Ejecutivo, sino que solo la administración de las prisiones sea de este, y se facultara al Poder Judicial mediante el nacimiento de la figura de los Jueces de Ejecución de Penas, siendo esta la autoridad judicial especializada competente para resolver controversias en materia de ejecución penal, así como garantizar la aplicación del debido proceso penitenciario, descartando las facultades de las autoridades penitenciarias y por ende judicializando con ella la etapa de ejecución.

Con esta división se dará a cada ámbito de poder lo que le corresponde: al Poder Ejecutivo, la administración de las prisiones y al Judicial la de ejecutar sentencias. Con esto la autoridad Judicial es decir los Jueces de Ejecución de Penas serán los encargados de vigilar el cumplimiento de las sentencias, que no solo implica la prisión como tal, sino que en el transcurso de estas es necesario la supervisión de una autoridad que pueda resolver respecto a situaciones tales como la aplicación de penas alternativas de prisión, la concesión de beneficios o el lugar donde se deba extinguir la pena.

Por otra parte, otro punto importante a resolver era la falta de capacitación del personal respecto a las áreas de psicología criminal, psiquiatría, penitenciarismo, victimología, estudio sistematizado de los documentos internacionales suscritos por México en materia de los derechos humanos de los sentenciados y su forma de trato y tratamiento, estadística y ejecución penal. El cambio de la denominación de reo por sentenciado por considerarse infame y denigrante, uno de los puntos de esta reforma penal es que su finalidad de que sea garantista cuidando y salvaguardando los derechos humanos de todas las personas independientemente que se encuentren privadas de la libertad por la comisión de un delito.

Otro concepto que se sugiere cambiar es el de readaptación por reinserción, puesto que se considera que la readaptación social es inadecuada para nombrar al momento en que los sentenciados dan cumplimiento a sus 
condenas y se insertan nuevamente a su entorno social en que vivían. Si se toma como referencia que la esencia de la prisión, como una institución total y excluyente, en donde se mantiene con una visión segregativa de la persona privada de la libertad, más que una intención de inclusión en la sociedad de dónde provenía. Considerando de esta forma se cambie por el término de Reinserción teniendo como objetivo el procurar que los reclusos no vuelvan a delinquir y brindarle ayuda para que se transforme en un ser humano que sea de utilidad a la sociedad al momento de dejarlo en libertad, es decir regresar a la persona que desprendimos de la sociedad por cometer un delito y reinsertarlo porque son parte de una sociedad procurando con ello no reincidan aplicando los sistemas de tratamiento basados en el trabajo, la educación el deporte y la salud.

Respecto a las personas que cometan delitos de delincuencia organizada se juzga conveniente prohibir que puedan compurgar sus penas en los centros penitenciarnos cercanos a su domicilio y por consecuencia sean internados en centros de reclusión especiales para estos internos. Se establece además la restricción a las comunicaciones de estas personas privadas de la libertad con terceros, salvo con su defensor e imponer medidas de seguridad especial dada su alta peligrosidad. De esta forma se plantea un fin u objetivo central que es "lograr la reinserción del sentenciado a la sociedad y procurar que no vuelva a delinquir".

Como medios para obtener este propósito central se plantean cinco ejes o bases para el logro de la reinserción social del sentenciado. Estos cinco ejes se desprenden del artículo 18 Constitucional en el segundo párrafo donde se establece el Sistema Penitenciario se organizará sobre la base del respeto a los derechos humanos, del trabajo, la capacitación para el mismo, la educación, la salud, y el deporte como medios para lograr la reinserción del sentenciado a la sociedad y procurar que no vuelva a delinquir, observando los beneficios que para él prevé la ley.

1. El respeto a los derechos humanos

2. El trabajo y capacitación para el trabajo

3. La educación

4. La Salud

5. El deporte 
Por otra parte, hay diversas disposiciones constitucionales que consagran Derechos fundamentales de las personas privadas de su libertad y que constituyen mandatos y directrices para el sistema penitenciario. Las directrices a las que se hace referencia son:

- Control judicial sobre la ejecución de las penas (artículo 21, pfo $\left.3^{\circ}\right)$

- Separación entre internos procesados y sentenciados, siendo recluidos en sitios distintos (artículo 18, pfo. $1^{\circ}$ )

- Posibilidad de que mexicanos que cumplan sus sanciones en otros países, sean trasladados a México para compurgar su condena con base en los sistemas de reinserción social (artículo 18, pfo. $7^{\circ}$ )

- Posibilidad (con las restricciones que establezca la ley) de cumplir la condena "en los centros penitenciarios más cercanos a su domicilio, a fin de propiciar su reintegración a la comunidad como forma de reinserción social" (artículo 18 pfo. 81).

- La prisión preventiva no podrá exceder del tiempo como máximo de pena fije la ley al delito que motivare el proceso y en ningún caso será superior a dos años, salvo que su prolongación se deba al ejercicio del derecho defensa del imputado... (artículo 20, B Fr. IX, pfo. 2)

- En toda pena de prisión que imponga una sentencia, se computará el tiempo de la detención (art. 20, B, Fr. IX, pfo $3^{\circ}$ ).

Con algunas adecuaciones instrumentales, hoy se aplica el modelo técnico- progresivo en el sistema penitenciario mexicano. Asociado a factores de valoración objetiva para el mejoramiento de perfil y la conducta de los internos sentenciados (Patiño, 2010:19)

\section{Ley Nacional de Ejecución de Penas}

Ley publicada en el Diario Oficial de la Federación el 16 de junio de 2016, la cual en su artículo $1^{\text {a }}$ hace referencia el objeto es establecer las normas que deben de observarse durante el internamiento por prisión preventiva, en la ejecución de penas y en las medidas de seguridad impuestas como consecuencia de una resolución judicial, así como el establecer los procedimientos para resolver las controversias que surjan con motivo de 
la ejecución penal y regular los medios para lograr la reinserción social. Se hace referencia de esta Ley en virtud de que anteriormente cada entidad federativa en nuestro País tenía su propia Ley que regulaba y establecía todas las facultades tanto de las autoridades involucradas en la reinserción social de los internos como los mecanismos para lograr la reinserción social en cada uno de los diferentes Estados de la República Mexicana.

Las controversias es un término nuevo que aparece en esta Ley en el Capítulo IV referente a las controversias mismas que se presentarán ante el Juez de Ejecución, y se habla de las situaciones en las cuales una persona privada de la libertad podrá poner a consideración del Juez de Ejecución y resuelva a su favor, en relación a las condiciones de internamiento, la modificación, extinción de las penas, derechos que tiene la persona privada de la libertad en relación a sus visitas, permisos, traslados, así como de las medidas de seguridad, mismas que se resolverán mediante un procedimiento ante la autoridad judicial. Anteriormente estas peticiones se realizaban ante la autoridad penitenciaria representada en el Comité Técnico Interdisciplinario y era esta autoridad quien atendía a las peticiones del interno.

En cuanto a los principios lo que se establece es que el desarrollo de los procedimientos en el Sistema Penitenciario se busque respetar estos principios para no violentar los derechos de todas las personas privadas de la libertad, en donde reciban un trato digno sin discriminación ya que es responsabilidad de las autoridades velar por que se respeten los derechos de las personas sujetas a esta ley y lograr la reinserción social del interno.

Autoridades corresponsables serán, las Secretarías de Gobernación, de Desarrollo Social, de Economía, de Educación Pública, de Cultura, de Salud, del Trabajo y Previsión Social y la Comisión Nacional de Cultura Física y Deporte, el Sistema Nacional para el Desarrollo integral de la Familia y la Secretaría Ejecutiva del Sistema Nacional de Protección Integral de Niñas, Niños y Adolescentes o sus equivalentes en las entidades federativas.

Anteriormente no existía esta figura de Policía Procesal propiamente ya que solo existía el personal que se encargaba de la seguridad de los centros como tal, mas sin embargo de momento para subsanar y dar cumplimiento con la seguridad de las personas privadas de la libertad en las Salas de Juicio Oral, fue de que del personal existente en los establecimientos penitenciarios los habilitaron como Policías Procesales, que esto ha tomado un reto porque 
las facultades que tienen los servidores públicos son muy diferentes a las tareas desempeñadas como Custodios en los Centros Penitenciarios.

La conferencia Nacional dictará los protocolos que serán observados en los Centros Penitenciarios., los cuales pretenden unificar los trabajos que se desarrollen en las 32 entidades federativas de nuestro País.

Cabe señalar que esta Ley consta de 207 artículos en donde principalmente se busca el mejoramiento de las condiciones de las cárceles en el País, ya que se quiere restablecer el funcionamiento y unificar criterios, se resaltan los siguientes aspectos que se muestran a continuación:

- Establecer normas que se deben observar durante el internamiento por prisión preventiva.

- Procedimientos para resolver controversias que surjan con motivos de la ejecución penal.

- Principios del Sistema Penitenciario: Igualdad, legalidad, debido proceso, transparencia, confidencialidad, reinserción social, dignidad.

- La autoridad penitenciaria promoverá que los Centros Penitenciarios sean sustentables.

- Deberá existir coordinación institucional, es importante señalar que se faculta para que autoridades denominadas corresponsables coadyuven con el cumplimiento de esta Ley, Figuera nueva que aparece en este ordenamiento.

- Derechos de las personas privadas de la libertad trato digno, asistencia médica, alimentación nutritiva, permanecer en estancias dignas, ser informado de derechos y obligaciones, suministro de agua potable, artículos de aseo, visitas, efectuar peticiones o quejas por escrito, plan de actividades, integridad física moral, sexual y psicológica, beneficiando a hombres, mujeres, indígenas, personas de la tercera edad, discapacitadas, con excepción de quienes hayan cometido delitos de delincuencia organizada, personas sujetas a medidas especiales.

- Se establecen los derechos y obligaciones a que quedan sujetas las personas privadas de la libertad. 
- Las autoridades del procedimiento de ejecución penal son: Autoridad penitenciaria, Custodia Penitenciaria, Titular de los centros penitenciarios.

- Comité Técnico integrado por: Administrativo, Jurídico, Técnico y de Custodia Penitenciaria.

- Policía Procesal, realiza traslados y da seguridad y custodia a la persona privada de la libertad.

- La actividad que se realice dentro de los centros penitenciarios será reglada por Protocolos de Actuación que deberán de acatar todas las autoridades responsables de los procedimientos de la ejecución penal, para efecto de garantizar las condiciones de internamiento dignas y seguras para la población privada de la libertad y la seguridad y bienestar del personal y otras que ingresan a los Centros, estos lineamientos se establecen en el artículo 34 de la Ley Nacional de Ejecución Penal.

- Respecto a las medidas de vigilancia especial, para las personas privadas de la libertad por delincuencia organizada o por medidas especiales se establece compurgarán sus penas en espacios especiales. Estas personas quedarán sujetas a cambio de dormitorio, módulo, estancia, vigilancia permanente, visitas periódicas, restricción de visitas familiares e intimas

- En cuanto a las faltas y sanciones serán establecidas por el comité técnico y estas deberán debidamente justificadas salvaguardando los derechos de las personas privadas de la libertad aislamiento temporal no deberá ser mayor a quince días continuos

- El procedimiento administrativo deberá presentarse ante la Autoridad Penitenciaria y el Procedimiento de ante el Juez de Ejecución

- Beneficios preliberacionales, y sanciones no privativas de la libertad: Beneficios de libertad anticipada, Libertad Condicional, Medidas penales no privativas de la libertad, Justicia Terapéutica, Justicia restaurativa, Mediación Penitenciaria.

Con la implementación de esta Ley de Ejecución Penal se pretende que a las personas privadas de la libertad se les respeten sus derechos humanos, 
pero además que las autoridades encargadas de la reinserción social se conduzcan bajo rubros de respecto y trato digno hacia las personas privadas de la libertad, que el actuar sea bajo lineamientos legales previamente establecidos en donde se marque la pauta de su actuar pero sobre todo que estos lineamientos sean unificados en las 32 entidades federativas de nuestro País y por ende implementados en los centros penitenciarios existentes.

Esta Ley busca subsanar todas las deficiencias que hasta la fecha se han venido dando dentro del Sistema Penitenciario y que de alguno u otra forma no ha cumplido su objetivo el que realmente se pueda dar una reinserción social en las personas privadas de la libertad. Ahora bien es de reciente creación por lo que a la fecha las autoridades encargadas de ejecutar no ha sido posible totalmente la implementación de esta ley, tal es el caso que dentro del mismo ordenamiento legal una vez entrado en vigencia se establecieron dentro de sus artículos transitorios prórrogas para el cumplimiento de lo que de la misma se deriva, estos plazos van desde un año o dos años después de la publicación para efecto de dar cumplimiento, sobre todo lo relacionado con infraestructura penitenciaria, y es razonable puesto que se tendrían que otorgar los presupuestos económicos necesarios para hacer los ajustes y otorgar los servicios que se establecen a las personas privadas de la libertad, por otra parte se establecen plazos de cuatro años a fin de que la autoridad penitenciaria capacite y adecue los establecimientos penitenciarios y su capacidad instalada, equipar desarrollar tecnologías de la información y comunicaciones, así como adecuar su estructura organizacional.

Cabe mencionar que a la fecha se siguen implementando estrategias para que cada Centro Penitenciario en relación a su personal se capacite y conozca los lineamientos que para su trabajo a desarrollar sea apegado de acuerdo a las normas aplicables para este caso en particular.

\section{Tratados Internacionales aplicables al funcionamiento y regulación del Sistema Penitenciario}

Ahora bien, como se señala en el artículo primero de la Constitución en donde se establece que la interpretación de las normas relativas a los Derechos Humanos se hará con esta Constitución y con los tratados internacionales de la materia favoreciendo en todo tiempo a las personas la 
protección más amplia". De esta forma las personas privadas de su libertad, que son los grupos más vulnerables frente al abuso de poder y la violación de Derechos Humanos, podrán disponer de una esfera más amplia de derechos y garantías.

Se puede referir, entre los instrumentos internacionales en los que se enuncian o desarrollan los derechos de las personas privadas de su libertad los siguientes: La Declaración Universal de los Derechos Humanos, la Declaración Americana de Derechos Humanos, el Pacto Internacional de Derechos Civiles y Políticos, las Reglas mínimas para el Tratamiento de los Reclusos, las Reglas mínimas de las Naciones Unidas sobre las personas privadas de la libertad, Reglas de Mandela, así como las resoluciones de multilaterales de protección a los Derechos Humanos.

La reforma a nuestro Sistema Penal trajo consigo grandes cambios los cuales hoy en día se siguen capacitando y subsanando errores que se han cometido en el transcurso de la implementación de este nuevo ordenamiento legal aplicable al Sistema Penitenciario.

\section{Población interna de los centros penitenciarios en México}

La población interna en los Ceresos, Ceferesos, Prisiones Militares y cárceles municipales que conforman la muestra asciende a 186,829 personas, lo que representa al $86.16 \%$ del total de la población penitenciaria nacional, población total penitenciaria a enero de 2017: 216,831, dato obtenido del Cuaderno mensual de información estadística penitenciaria nacional, publicado por la Secretaría de Gobernación, correspondiente a enero de 2017.

Tomando en referencia que la Comisión Nacional de los Derechos Humanos, como parte de sus atribuciones, realiza el Diagnóstico Nacional de Supervisión Penitenciaria (DNSP) con el objetivo de observar el respeto de los derechos humanos de las personas privadas de la libertad de forma anual y publica los resultados obtenidos con la finalidad de que las observaciones realizadas por esta autoridad se tomen en cuenta para las mejoras del propio sistema penitenciario.

El personal de la CNDH visita a cada uno de los centros penitenciarios con la finalidad de supervisar, entrevistar y realizar su reporte en base a los siguientes puntos: 
1. Aspectos que garantizan la integridad física y moral de las personas privadas de la libertad;

2. Aspectos que garantizan una estancia digna;

3. Condiciones de gobernabilidad;

4. Reinserción social del interno; y

5. Grupos de internos con requerimientos específicos.

Dentro de estos cinco rubros permiten revisar los indicadores mismos que se califican en una escala del 0 al 10, de acuerdo con las condiciones mínimas que debe de existir en un centro penitenciario, estas visitas se hacen de forma anual a todos los centros penitenciarios del País. La calificación nacional de Ceresos en 2017 es de 6.30, con lo cual se tiene una calificación a nivel general mayor que en los últimos tres periodos evaluados.

El levantamiento del DNSP 2017, se realizó sobre una muestra total de 198 instituciones penitenciarias de las 378 que se encontraban en funcionamiento al inicio de 2017, el dato de 378 instituciones penitenciarias se conforma de los 375 centros registrados en el Cuaderno mensual de información estadística penitenciaria nacional, correspondiente a enero de 2017, más las 3 Prisiones Militares que existen en el país con lo cual se abarcó más de la mitad de los centros de reclusión del país. La muestra considerada para centros penitenciarios dependientes de gobiernos locales (varoniles, mixtos y femeniles) fue de 131, es decir el $47.81 \%$ de un total de 2744 existentes en el territorio nacional, la población privada de la libertad en esos establecimientos fue de 161,855 personas, lo que corresponde al $84.75 \%$ del total de la población recluida en centros estatales, cuyo texto íntegro del Diagnóstico Nacional de Supervisión Penitenciaria 2017 puede consultarse en el siguiente enlace: http://www.cndh.org.mx/sites/all/doc// sistemas/DNSP/DNSP_2017.pdf.

La muestra considerada para centros penitenciarios dependientes de gobiernos locales (varoniles, mixtos y femeniles) fue de 131, es decir el $47.81 \%$ de un total de 2744 existentes en el territorio nacional, la población privada de la libertad en esosestablecimientos fue de 161,855 personas, lo que corresponde al $84.75 \%$ del total de la población recluida en centros estatales. Con relación a las cárceles que dependen de autoridades municipales, 5 se consideró una muestra de 44 (52.38\%) establecimientos de un total de 84 
(67 municipales y 17 distritales) que había en enero de 2017, la población que se encontraba interna en los centros supervisados era de 2,529 internos, lo que representó un $75.08 \%$ del total de la población recluida en ese tipo de instituciones. Se visitaron los 20 centros federales en funcionamiento, al considerar el Cefereso CPS $N^{\circ} 17$, en Michoacán que inicio actividades en 2017, el Centro Federal de Rehabilitación Psicosocial (CEFEREPSI) y los 4 establecimientos que conforman el Complejo Penitenciario de Islas Marías, con una población total de 21,906 personas privadas de la libertad al momento de las visitas. También se incluyen las tres Prisiones Militares, mismas que albergaban durante la supervisión a 539 internos.

El DNSP 2017 contó con la participación de 21 servidores públicos adscritos a este Organismo Nacional y el apoyo de personal de las Comisiones locales de Derechos Humanos de todas las Entidades, quienes aplicaron las "Guías de Supervisión" que sirvieron de base para la evaluación de los cinco rubros que reflejan las condiciones que existen en las prisiones supervisadas, entre las que destacan las siguientes:

\section{Centros Estatales en México en 2018}

- Las deficiencias detectadas con mayor incidencia se refieren a la separación entre procesados y sentenciados, deficientes condiciones materiales, equipamiento e higiene de las áreas de dormitorios, sancionados, sujetos a protección, cocina y comedores, insuficiente personal de seguridad y custodia, hacinamiento, falta de actividades laborales y de capacitación para el trabajo. También se identificaron como deficiencias importantes con 71 y 69 respectivamente, la falta de prevención y atención de incidentes violentos, así como las condiciones de autogobierno/cogobierno.

- En la mayoría de los centros visitados no se cuenta con algún programa para la prevención de adicciones y de desintoxicación voluntariaeficiente, prevaleciendo deficienciascomolainexistencia o insuficiencia de instalaciones necesarias para el funcionamiento del Centro.

- En 69 centros visitados se pudo observar un bajo control en el ejercicio de las funciones de autoridad por parte de los servidores 
públicos, en actividades productivas, educativas y deportivas, de alimentación, mantenimiento y limpieza, así como el control del ingreso de visitas tanto familiar como íntima, uso de los teléfonos y de la seguridad, dando paso al autogobierno/cogobierno, situación que vulnera el control que debe tener la autoridad sobre el Centro, favoreciendo la violencia dentro del mismo.

- En 46 de los centros estatales persiste la sobrepoblación, lo que genera otro tipo de problemáticas que afectan su funcionamiento. En 64 de los establecimientos locales supervisados existe hacinamiento, con áreas que rebasan de manera importante su capacidad.

- En la mitad de los centros verificados, se observaron áreas de privilegios, así como la presencia de objetos y sustancias prohibidas.

En el documento del Diagnostico la calificación más alta la obtuvieron los Estados de Guanajuato y Querétaro con una puntuación de 8.19 y 8.13 respectivamente, siendo que la calificación más baja la obtuvieron Guerrero con 4.22 y Nayarit 4.15 .

\section{Centros Federales}

- En 16 establecimientos hay insuficiencia de personal, en 14 concurre la carencia de actividades laborales y de capacitación; así como de actividades educativas en 6 de los establecimientos.

- El derecho de protección de la salud es un problema generalizado en los centros federales dada la falta de personal médico, así como por la deficiencia en su atención.

- En 11 centros se observaron deficiencias en la atención a personas adultas mayores y en 13 en los programas para la prevención de adicciones y desintoxicación voluntaria.

- En 6 de los centros federales se detectó falta de acceso de las personas privadas de la libertad en actividades deportivas.

- En 11 de los establecimientos federales se observaron deficiencias respecto de la vinculación de las personas privadas de la libertad con la familia y sociedad, así como en 6 centros se observaron deficiencias en la prevención de incidentes violentos. 
- Se detectó en 8 centros deficiente clasificación entre procesados y sentenciados. Diagnóstico Nacional de Supervisión Penitenciaria 2017

- Centros Municipales

- En lo que respecta a centros municipales, las deficiencias detectadas con mayor incidencia se refieren a insuficientes actividades laborales y de capacitación para el trabajo, así como una deficiente clasificación, falta de reglamentos y manuales, así como escasa o a veces nula difusión de la normatividad que rige al Centro hacia las personas privadas de la libertad y no se cuenta con algún programa para la prevención de adicciones y de desintoxicación voluntaria.

- Por lo que se refiere a los servicios para mantener la salud de las personas privadas de la libertad, la mayoría de los centros carecen de instrumental médico, medicamentos y de personal suficiente para atender a las personas. También se observó insuficiencia o inexistencia de instalaciones necesarias para el funcionamiento del Centro, así como de acciones para prevenir y atender incidentes violentos.

- Parte importante son las malas condiciones materiales e higiene de las instalaciones para alojar a las personas privadas de la libertad, deficienteintegración delosexpedientestécnico-jurídicos, asícomo deficiencia en agua y alimentos además de su pésima elaboración y distribución.

De las reformas que se hacen a nuestro sistema penal en cual va incluido el Sistema Penitenciario, se busca establecer los lineamientos para que se retome la finalidad de la prisión que es la reinserción social de las personas privadas de la libertad, considero que es un gran paso con la reforma debido a que era urgente se alineara el rumbo de nuestros Sistema Penitenciario, porque realmente no se había dado cumplimento a su encomienda. Y sin duda alguna dentro de los aspectos a considerar en esta reforma son precisamente para dar solución a todos los problemas que hoy en día se viven en los centros penitenciarios.

Si es necesario recalcar que solo son dos años de la reforma y de que entraron en vigor las leyes aplicables y que aún a la fecha no se han visto resultados al cien por ciento porque en la mayoría de los casos se 
ha detenido, puesto que no se ha destinado el recurso económico para que en algunos de las instituciones penitenciarias se modifique las estructura para que se construyan los espacios de acuerdo a la LNEP, como lo es las estancias infantiles que deben tener los centros que albergan mujeres que cuenten con hijos, puesto que tiene derecho a tenerlos con ellas hasta la edad de cinco años, así como la contratación de todo el personal necesario para dar la atención que requiere un menor de edad cuidando desde su salud y su educación, esparcimiento y áreas de recreación dentro del establecimiento penal.

Por otra parte, el personal penitenciario en cada uno de los establecimientos es mínimo al que debiera de estar para brindar los servicios adecuados, así como lo marca la LNEP, no se había dado la importancia porque no se profesionaliza ni se actualizaba al personal, además de que las condiciones de trabajo dejan mucho que desear, con la reforma se ha dado un poco de capacitación y las autoridades se han dado cuenta de la importancia de la actualización en su personal. Hay desconocimiento sobre todo en los instrumentos internacionales, su forma de aplicación y que son fundamentales para el trabajo diario que realizan.

\section{Conclusiones}

Si se logra respetar la capacidad instalada para cada uno de los establecimientos se lograría proporcionar todos los servicios a las personas privadas de la libertad como lo es un trato digno, la alimentación, la salud, la educación, capacitación para el trabajo y respeto a los derechos humanos que son los cinco ejes en el cual el Sistema Penitenciario se basa, además se evitaría el hacinamiento y por ende se impedirían disturbios tales como el autogobierno y finalmente se lograría la reinserción social de las persona privadas de la libertad. 
Un problema que se ha presentado es que a la fecha no se tienen la totalidad de Protocolos de actuación de los Macro procesos que rigen el Sistema Penitenciario y que son vitales para cada una de las actividades que desarrolla el personal penitenciario teniendo como finalidad salvaguardar los derechos humanos de todas las personas privadas de la libertad, no se han puesto de acuerdo ya que esos protocolos al igual que la LNEP deberán e ser de observancia general para todos los Centros Penitenciarios del país, siendo de conocimiento propio de los autores que, a la fecha se siguen trabajando con la construcción de los protocolos de actuación porque entre las mismas autoridades se ha dificultado ponerse de acuerdo en cómo realizan sus funciones y que además deben de cuidar que estos sean claros, se han hechos varias revisiones y se han hecho modificaciones, pero no se han definido en un cien por ciento, esta tarea es propia además de la Conferencia Nacional del Sistema Penitenciario.

El Diagnóstico que presenta la Comisión Nacional de Derechos Humanos es un documento que permite hacer las observaciones a las autoridades competentes para que traten de atender las necesidades que se presentan en cada uno de los establecimientos penitenciarios, es una tarea que apenas comienza aunado a la reforma constitucional, considerando que es un reto, pero sin duda alguna se tendrá éxito si cada uno de las instituciones, operadores del sistema y personal penitenciario trabajan en conjunto se logrará la Reinserción Social.

\section{Referencias}

Arias, J. P. (2010). Nuevo modelo de Administraciòn penitenciaria. Mèxico: Porrùa.

Bremauntz, E. M. (2004). Derechos Penitenciario. Mèxico: Mc Grw Hill.

Documenta. (01 de Agosto de 2018). Observatorio de prisiones. Obtenido de Observatorio de prisiones: http://observatorio-de-prisiones.documenta.org.mx/archivos/1475

INEGI. (01 de Agosto de 2018). Censo Nacional de Gobierno, Seguridad Pública y Sistema Penitenciario Estatales 2017. Obtenido de Censo Nacional de Gobierno, Seguridad Pública y Sistema Penitenciario Estatales 2017: http://www.beta.inegi.org.mx/app/biblioteca/ficha.html?upc $=702825095413$ 
Leucona, G. Z. (01 de agosto de 2018). Mèxico Evalùa. Obtenido de Mèxico Evalua.

Martìnez, G. M. (2007). Derecho Penitenciario Prisiòn y Control Social. Mèxico: Flores Editor y Distribuidor, S.A. de C.V.

México, C. N. (01 de Agosto de 2018). Diagnóstico Nacional de Supervisión Penitenciaria 2017. Obtenido de Diagnóstico Nacional de Supervisión Penitenciaria 2017: http://www.cndh.org.mx/sites/ all/doc/sistemas/DNSP/DNSP_2017.pdf

Paz, M. (2008). Derecho Penitenciario. Mèxico: Oxford. 